\title{
Impact of COVID-19 pandemic on Paediatric Residency Training Programme in Sudan: Challenges and the Way Forward
}

Inaam N Mohamed ( $\square$ inaamgashey@gmail.com )

University of Khartoum

\section{Safaa Nasr}

University of Khartoum

Ilham M Omer

University of Khartoum

\section{Research Article}

Keywords: COVID-19, residency, program, paediatrics, Sudan

Posted Date: May 14th, 2021

DOI: https://doi.org/10.21203/rs.3.rs-478149/v1

License: (c) (i) This work is licensed under a Creative Commons Attribution 4.0 International License.

Read Full License 


\section{Abstract}

Background: The clinical education and hands-on training are affected by the disruption of formal health care delivery and the emergency need to adapt the new rapidly evolving Covid 19 pandemic .Here we aim to determine the impact of COVID-19 paediatric on residency program training in Sudan.

Methods: This study was carried out in the period from December 2020 - January 2021. A designed questionnaire was distributed using Google form, 186 trainees responded.

Results: The study included 186 trainees of whom 152 (83.5\%) females and 30(16.5\%) males. Of whom 15 (8.2\%) acquired infection with Covid 19. Comparing the work load and teaching opportunities in the year 2019 to 2020 it was very clear that there is decrease in working load and training opportunities during Covid 19 pandemic. Almost all trainees fully agreed that their training was severely affected during Covid 19 pandemic locked down. Trainees raised the issue of being stressed by transmitting the infection to their families and the working environments.

Conclusion: The undue effect of the COVID-19 pandemic on residency paediatric training is well documented. Different innovative alternative teaching methods must be implemented to maintain quality of education, and research.

\section{Introduction}

Covid-19 is the fastest evolving and most horrific pandemic in the recent global history. It is perhaps the most daunting challenge humanity has faced since World War. ${ }^{1}$ The WHO declared coronavirus disease 2019 (COVID-19) a global pandemic on 11 March 2020 and Sudan was one of the most exposed countries in Africa, Khartoum (the capital) was the most affected region. ${ }^{2}$ The first case in Sudan was reported on 13 March 2020, and up to 28/ April 2021 there are 33,104 positive Covid 19 cases and 2,349 deaths. ${ }^{3}$ The government of Sudan has implemented preventive measures during the coronavirus disease pandemic, such as partial lockdown, contact monitoring, risk communication, social distance, and isolation to prevent the spread of Covid 19 virus. ${ }^{4}$ These measures had its impact on health system and on training and education in general. We believe that COVID-19 pandemic has significantly impacted the pediatric residency education and training, and profoundly changed the daily practice in Sudan. A number of operational changes, including modified clinical and didactic training policies, have been implemented to mitigate the spread of COVID-19 worldwide. ${ }^{5}$ The mitigation measures ${ }^{6}$ also advised reduction of doctor and patient contact. A direct consequence of this measure is the postponement of outpatient's clinic appointments, resulting in a decline in clinical exposure. The academic medical institutions confronted with a unique set of challenges with respect to ensuring the safety and well-being of resident versus providing service to patients and continue training program quality.

In this study we aim to study the impact of Covid 19 pandemic on pediatric residency training program and to compare training opportunities before(2019) and during(2020) Covid 19 pandemic in Sudan. We 
as well address the trainees' perception towards training and working environment during Covid 19 pandemic.

\section{Methods}

This is a comparative, cross sectional facility based study. This study was conducted during the period from January to February 2021. It was led at the SMSB including five paediatric training centres:

Omdurman, Bahri, Soba, Khartoum and Wad Medani. The study population were final-year trainees, who completed 4 years training according to the updated Paediatric and child health residency program curriculum (2020).

\section{Pediatric child health residency program Structure}

The Sudanese Medical Doctorate (MD) for the Paediatrics residency curriculum (revised 2020) is a competency based postgraduate programme held in Sudan under the umbrella of the Sudan Medical Specialization Board (SMSB). SMSB is the sole professional training body in Sudan mandated to manage and deliver medical and health specialty programs in the country. The program adopts major trends and reforms in medical education and graduate training regionally and globally. The main goal of this curriculum is emphasis on education, service and research together with the obligation of pediatricians to public health, professionalism, team work \& leadership and social accountability. The duration of the programme is 4 years with promotion exams in between the training years. The program is structured with different training opportunities, ranging from primary to tertiary healthcare levels. The program adopts a variety of teaching/learning methods with emphasis on Student-centered, Problembased and Community-based learning. The rotations cover general pediatrics, primary health care and pediatric subspecialties.

\section{Components and competencies}

Pediatrics and child health Residency Program is guided by three main administrative components inclusive of clinical experience, didactic teaching, and duty hours. Six core competencies have been identified to guide the design of a well-balanced pediatrics training program. These competencies include Professional values, attitudes, behavior and ethics, scientific foundation of medicine, clinical skills, communication skills, Critical thinking and research, Management of information and Population health and health system

The current number of trainees joining the program is approximately 1000 , of whom 300 in the final year (R4).

Sampling: Using a designed questionnaire, which was distributed to pediatric trainees in training using Google form. One hundred and eighty six responded with a response rate of $62 \%$. The questionnaire consist of questions related to training opportunities before and after Covid 19 pandemic and its impact on training. 


\section{Statistical analysis}

Data were analyzed using SPSS software version 23.0. Regarding descriptive statistics, categorical variables are presented as numbers and percentages. The recorded patient visits in 2019 and 2020 were compared using Pearson's chi-square or Fisher's exact tests for categorical variables. A p value of $<0.05$ was considered statistically significant.

\section{Results}

\section{Demographic data}

The study included $152(83.5 \%)$ females and 30(16.5\%) males. The five group for discussion included 30 candidates, 5-7 in each group, of whom 20(66.7\%) were females and 10(33.3\%) were males. The mean age was (30.38 \pm 3.23$)$ years. The trainees representing all academic tract, the majority from Soba Tract 74(40.7\%), Omdurman 52(28.6\%), Bahry31 (17.0\%), Khartoum 19(10.4\%) and six (3.3\%) are from Medani tract. One hundred and forty two $(78.0 \%)$ were at Khartoum State at the time of the Covid pandemic while $40(22.0 \%)$ were outside Khartoum State. One hundred and fifty five (85.2\%) trainees were working full time, $21(11.5 \%)$ in vacation, two $(1.1 \%)$ freeze their rotation and three $(1.6 \%)$ had sick leave while one $(0.5 \%)$ was absent. Only four (2.2\%) trainees volunteered to work in Covid 19 isolation centers.

\section{Effect of Covid 19 on trainees' health and wellbeing}

Fifteen (8.2\%) had positive PCR for Covid 19 and they were all symptomatic, all recovered with home management protocol. One hundred and eight (59.3\%) had contacted patients, colleagues, family members with positive Covid 19 which required home isolation for at least 14 days.

\section{Trainees' concerns during Covid 19 pandemic}

During Covid 19 pandemic 120(65.9\%) trainees fully agreed that there is shortage of medical and paramedical staff and 125(68.7\%) fully agreed that there is scarcity of administrative staff especially in night shifts. Seventy (37.6\%) trainees fully agreed that measures taken by the hospital to mitigate Covid 19 spread such as availability of protection devices (mask and gloves) is not sufficient. Hundred (53.7\%) trainees reach their work place via public transportation which may increase risk of Covid 19 transmission. One hundred sixty two (87.0\%) mentioned that they were stressed about hazard of acquiring Covid 19 infection from hospital and hence transmit it to their elderly parents ,kids and other family members. One hundred seventy seven $(97.3 \%)$ trainees stated that their families were resistant to led them working in these risky environment.

\section{Work load and duties}

Comparing the work load and teaching opportunities in the year 2019 to 2020 it was very clear that there is decrease in working load and training opportunities during Covid 19 pandemic. The majority (53.3\%) of trainees in 2019 work on daily basis compared to $6.0 \%$ in 2020. (figure1) 
The majority $57.7 \%$ of trainees in the year 2019 tend to cover ward on call three to four times per months compared to $11.9 \%$ in the year 2020 (figure2 )

Sixty percent of trainees in the year 2019 cover emergency department on call four times per month compared to $8.2 \%$ in the year 2020 (figure3)

\section{Patients' exposure (encounter)}

Almost half $(50.3 \%$ ) of trainees in the year 2019 used to be exposed to more than 30 patients in the inpatient ward per month, while in the year 2020 the majority $50.5 \%$ exposed to less than 10 patients per month. (figure4)

$42.2 \%$ of trainees in the year 2019 were exposed to more than 50 patients in the outpatient department per month while in the year 2020 the majority $64.2 \%$ exposed to less than 20 patients per month. (figure5)

$44.5 \%$ of trainees in the year 2019 were exposed to more than 50 patients in the emergency department per month while in the year 2020 the majority $50.5 \%$ exposed to less than 10 patients per month. (figure6)

\section{Unit activities / Didactic teaching}

In the year 2019 the majority 170(93.4\%) of trainees had grand round twice a week compared to $32(17.6 \%)$ in 2020 , this is similar to other activities such as discharge clinic, teaching activities (Tutorial/seminar/case presentation / radiology sessions/ journal club ...etc.) and research work activities. Table 1 


\begin{tabular}{|c|c|c|c|c|}
\hline & \multicolumn{2}{|c|}{ Year 2019} & \multicolumn{2}{|c|}{ Year 2020} \\
\hline & Number & $\%$ & Number & $\%$ \\
\hline \multicolumn{5}{|c|}{ Grand round/ week } \\
\hline Once & 23 & 12.6 & 137 & 75.3 \\
\hline Twice & 137 & 75.3 & 32 & 17.6 \\
\hline More & 22 & 12.1 & 13 & 7.1 \\
\hline \multicolumn{5}{|c|}{ Unit discharge clinic } \\
\hline Weekly & 150 & 82.4 & 15 & 8.2 \\
\hline Monthly & 32 & 17.6 & 43 & 23.6 \\
\hline None & 0 & 0 & 124 & 68.1 \\
\hline \multicolumn{5}{|c|}{ Teaching activities (Tutorial/seminar/case presentation / radiology sessions ...etc.) } \\
\hline Weekly & 179 & 98.4 & 63 & 34.6 \\
\hline Monthly & 3 & 1.6 & 16 & 8.8 \\
\hline None & 0 & 0 & 103 & 56.6 \\
\hline \multicolumn{5}{|c|}{ Research work and supervision } \\
\hline Yes & 182 & 100 & 72 & 39.6 \\
\hline No & 0 & 0 & 110 & 60.4 \\
\hline
\end{tabular}

Table 1: Unit activity and teaching during the year 2019 compared to 2020

\section{Online activities}

Twenty two (14.5\%) trainees had regular online activities (tutorial/ case based discussion/ radiology meetings /.....ect) in 2020 compared to three (1.6\%) in 2019. Eighty one trainees (44.5\%) joined online courses/ webinars related to training on Covid 19 detection, management and triage protocols. Hundred (53.7\%) trainees joined webinars and online lectures related to pediatric and child health problems

One hundred and seventy three $(95.0 \%)$ of trainees had access to internet with good quality and $153(84.0 \%)$ had smart phones and laptops.

In discussion groups almost all trainees fully agreed that their training was severely affected during Covid 19 pandemic locked down.

\section{Discussion}


The impact of Covid 19 virus on children has been comparatively mild despite its overwhelming effects on adult populations globally. Among the affected children, most have mild symptoms and some are even asymptomatic $(7,8,9)$ The Chinese Centre for Disease Control and Prevention has reported that among $\sim 70,000$ cases, $<1 \%$ were aged $<10$ years. ${ }^{10}$ However measures taken to alleviate the spread of Covid 19 virus have transformed the current setting of paediatric practice and have thus impacted on our patients as well as our paediatric residency training. Several researchers worldwide claim the need to enhance medical training programs and comprehensive continuing medical education with an emphasis on skills and competencies development. ${ }^{11}$

In this study it was very obvious that encounters with inpatients are decreased and outpatient encounters are nearly absent which will definitely led to decrease in the opportunities to counsel on preventive health and managing chronic disease. These competencies are very important to be acquired by trainees if we take into consideration that in Sudan non-communicable diseases are increasing dramatically, at the same time, the burden of chronic and endemic infections disease remains considerable. ${ }^{12}$ In our set up and maybe other resource limited countries measures to adapt with these special situation and challenges during Covid 19 pandemic are extremely enormous due to poor infrastructure, inadequacy and fragility of the health systems. ${ }^{13} \mathrm{~A}$ lot of effort had been done by Ministry of Health, governmental and non- governmental organizations in Sudan to help communication with patients during Covid 19 locked down as well as training the trainees for triage and management protocol for Covid 19 infected patients and contacts. However very little had been done to adjust formal training of trainees and adaptive measures were not enough.

In this study $8.2 \%$ of the trainees had acquired Covid 19 virus infection fortunately all recovered with home management protocol. Not only the paediatricians but also other healthcare professionals are at a higher risk of infection with COVID-19. Golnar Sabetian et al reported a rate of 5.6\% infection among health care worker in southwest Iran, ${ }^{14}$ however in countries where Covid 19 infection rate is high such as Italy reported that $20 \%$ of healthcare professionals had become infected with Covid $19 .{ }^{15}$ In this study the majority of trainees fully agreed that measures taken by the hospital to mitigate Covid 19 spread were insufficient, which was similarly reported by Sarah et al form Sudan. ${ }^{16}$

Trainees in this study were stressed by transmission of Covid 19 infection to their families or acquiring infection. Lo $D$ reported that paediatricians, especially those who have children at home, not only have concerns about passing the infection to their children but also about not caring enough for their children during quarantine period, considering school closures and social distancing policies. ${ }^{17}$ Various psychological factors, including stress, anxiety and burnout, are extensively discussed in research studies emphasizing the need for integrated psychological intervention programs for the support of physicians and medical staff however these issues need further study among our population.

In this study it is evident that during Covid 19 pandemic the interactions of trainees with the trainers was interfered and most of the training activities were postponed. This ultimately affect the clinical 
competency and experiences of trainees. Trainees, therefore, may no longer meet their traditional graduation requirements. ${ }^{19}$ To anticipate the reduction in learning opportunities and to cope with the situation during Covid 19 pandemic a lot of academic institutions worldwide adopt innovative teaching methods. Danielle $\mathrm{G}$ et al from Department of Paediatrics, Boston Children's Hospital wrote about advocacy during the COVID-19 Pandemic. They stated that trainees have necessity to developed new ways to connect with patients and families to ensure their general well-being from afar. ${ }^{20}$

The COVID-19 pandemic has affected research activities to a great extent as reported in this study. As a direct consequence of lockdown measures and the closure of most university research facilities, all research activities unrelated to COVID-19 have been paused this was similarly reported in the literature. 21

As the majority of trainees in this study had access to internet and smart phones this will help establishing online training activities. There is a need to support training with technology-enhanced learning methods and active learning strategies that requires strategic support. Stakeholders need to take action in order to develop and implement innovative methods for medical training taking into consideration the current new norms and the setup in Sudan.

\section{Conclusion}

In order to continue training and to preserve the quality of the certificate it's important that the program will adapt an innovative teaching and training methods using advanced technology, simulation and online teaching.

\section{Abbreviations}

SMSB: Sudan Medical Specialization Board

MD: Medical Doctorate

\section{Declarations}

\section{Acknowledgements}

Our thanks and gratitude to all trainees in paediatrics who participated in this study

\section{Authors' contributions}

IN: Prepared the Concept paper, designed the questionnaire, filling and interview of Trainees, data analysis, drafted manuscript, publication process

SN: Filling the questionnaire, drafted and edited manuscript, Data entry and analysis. 
IO: filling the questionnaire and interview of trainees, Study design, Data entry and Data analysis

\section{Funding}

Not applicable

\section{Availability of data and materials}

The dataset used and analysed during the current study is available from the corresponding author on reasonable request.

\section{Ethics approval and consent to participate}

Ethical approval was obtained from Research and Ethical Committee at SMSB.

All methods are carried out in accordance with relevant guidelines and regulations. All the participants voluntarily participated in this study and provided written informed consent.

\section{Consent for publication}

Not applicable

\section{Competing interests}

The authors declare that they have no competing interests.

\section{References}

1. Hui DS, Azhar El, Madani TA, Ntoumi F, Kock R, Dar O, et al. The continuing 2019-Covid epidemic threat of novel coronaviruses to global health-the latest 2019 novel coronavirus outbreak in Wuhan, China. Int J Infect Dis 2020; 91:264-6.

2. Domenico Cucinotta, Maurizio Vanelli. WHO Declares COVID-19 a Pandemic. Acta Biomed. 2020; 91(1):157-160.

3. N.Altayb, N.M.E.Altayeb, Y.Hamadalnil , M.Elsayid, N.E.Mahmoud. The current situation of COVID-19 in Sudan. New Microbes and New Infections 2020; 37:100746.

4. https://www.worldometers.info/coronavirus/country/sudan /

5. Nasca T. ACGME's early adaptation to the COVID-19 pandemic: principles and lessons learned. J Grad Med Educ. 2020; 12:375-378.

6. Kamal Kant Sahu, Raman Kumar. Preventive and treatment strategies of COVID-19: From community to clinical trials. Journal of family medicine and primary care 2020; 9:2149-2157.

7. Dong Y, Mo X, Hu Y. Epidemiology of COVID-19 among children in China. Pediatrics 2020; 145(6):e20200702. 
8. Cruz A, Zeichner S. COVID-19 in children: initial characterization of the pediatric disease. Pediatrics 2020;145 (6) e20200834

9. Lu X, Zhang L, Du H. SARS-CoV-2 infection in children. N Engl J Med 2020;382(17):1663-1665

10. Wu Z, McGoogan JM. Characteristics of and important lessons from the coronavirus disease 2019 (COVID-19) outbreak in China: summary of a report of 72314 cases from the Chinese Center for Disease Control and Prevention. JAMA 2020 ;323(13):1239-1242.

11. Li, L.; Xv, Q.; Yan, J. COVID-19: The Need for Continuous Medical Education and Training. Lancet Respir. Med. 2020; 8: e23.

12. Esmita Charani, Aubrey J Cunnington, AlaEldin H A Yousif, Mohammed Seed Ahmed, Ammar E M Ahmed, Souad Babiker et al. In transition: current health challenges and priorities in Sudan. BMJ Glob Health. 2019; 4(4): e001723.

13. Ebrahim M. A. Ebrahim, Luam Ghebrehiwot, Tasneem Abdalgfar, Muhammad Hanafiah Juni. Health Care System in Sudan: Review and Analysis of Strength, Weakness, Opportunity, and Threats (SWOT Analysis). Sudan Journal of Medical Sciences.2017; 12(3):133.

14. Golnar Sabetian, Mohsen Moghadami, Leila Hashemizadeh Fard Haghighi, Reza Shahriarirad, Mohammad Javad Fallahi, Naeimehossadat Asmarian et al. COVID-19 infection among healthcare workers: a cross-sectional study in southwest Iran. Virology 2021; 18: 58.

15. Votto, Maria De Filippo, How pediatrics resident's life has changed during the COVID-19 pandemic. Italian Journal of pediatrics. 2020; 46:156.

16. Sarah Misbah El-Sadig, Lamis Ahmed Fahal, Ziad Bakri Abdelrahim, Eiman Siddig Ahmed, Nouh Saad Mohamed, and Emmanuel Edwar Siddig. Impact of COVID-19 on doctors and healthcare providers during the pandemic in Sudan.Trans R Soc Trop Med Hyg. 2021; 0: 1-2.

17. Lo D. COVID-19: protecting healthcare workers. Lancet 2020; 395(10228):922.

18. Dubey, S.; Biswas, P.; Ghosh, R.; Chatterjee, S.; Dubey, M.J.; Chatterjee, S.; Lahiri, D.; Lavie, C.J. Psychosocial .Impact of COVID-19. Diabetes Metab. Syndr. 2020, 14, 779-788.

19. Ahmed, H.; Allaf, M.; Elghazaly, H. COVID-19 and Medical Education. Lancet Infect. Dis. 2020; 20: 777-778.

20. Danielle G. Rabinowitz, Kathryn M. Sundheim. Trainee-Directed Educational Pursuits and Advocacy during the COVID-19 Pandemic. PEDIATRICS 2020; 146: e20201564.

21. Termini C.M., Traver D. Impact of COVID-19 on early career scientists: an optimistic guide for the future. BMC Biol. 2020; 18(1):95.

\section{Figures}




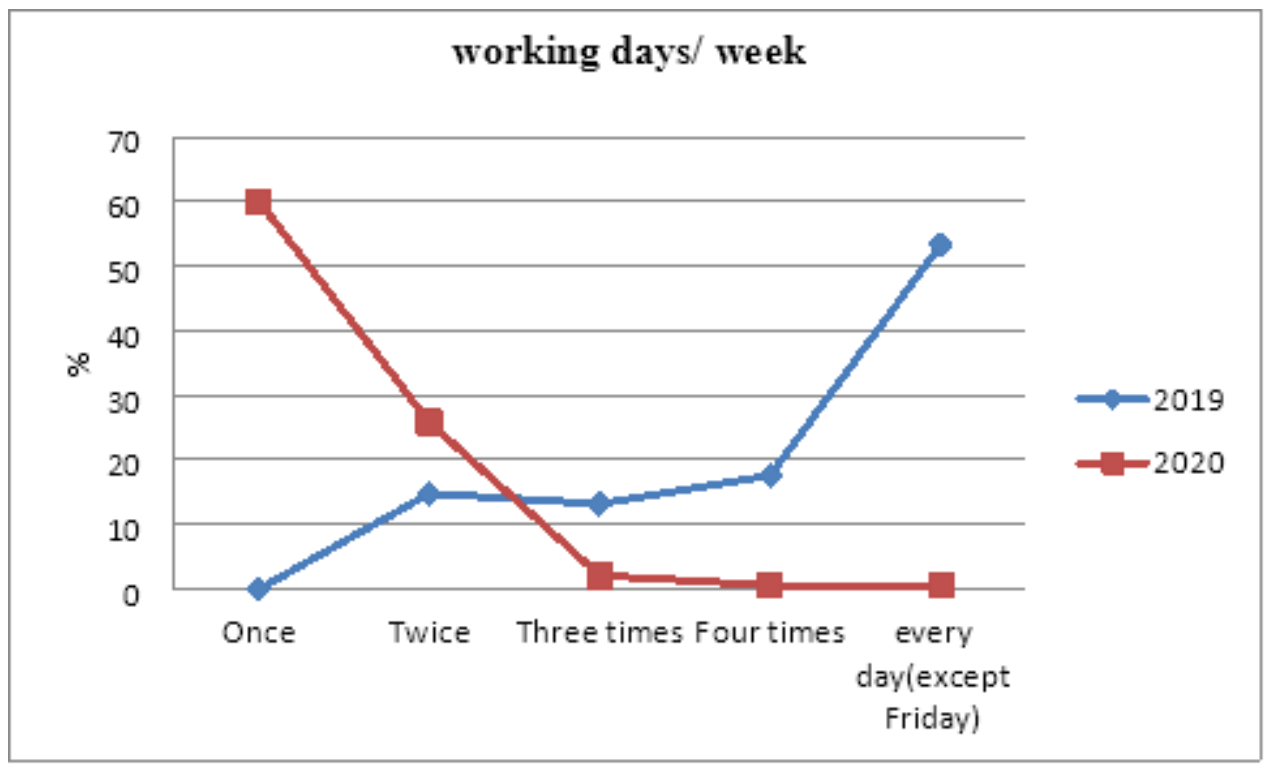

Figure 1

Trainees' working days per week in year 2019 compared to 2020

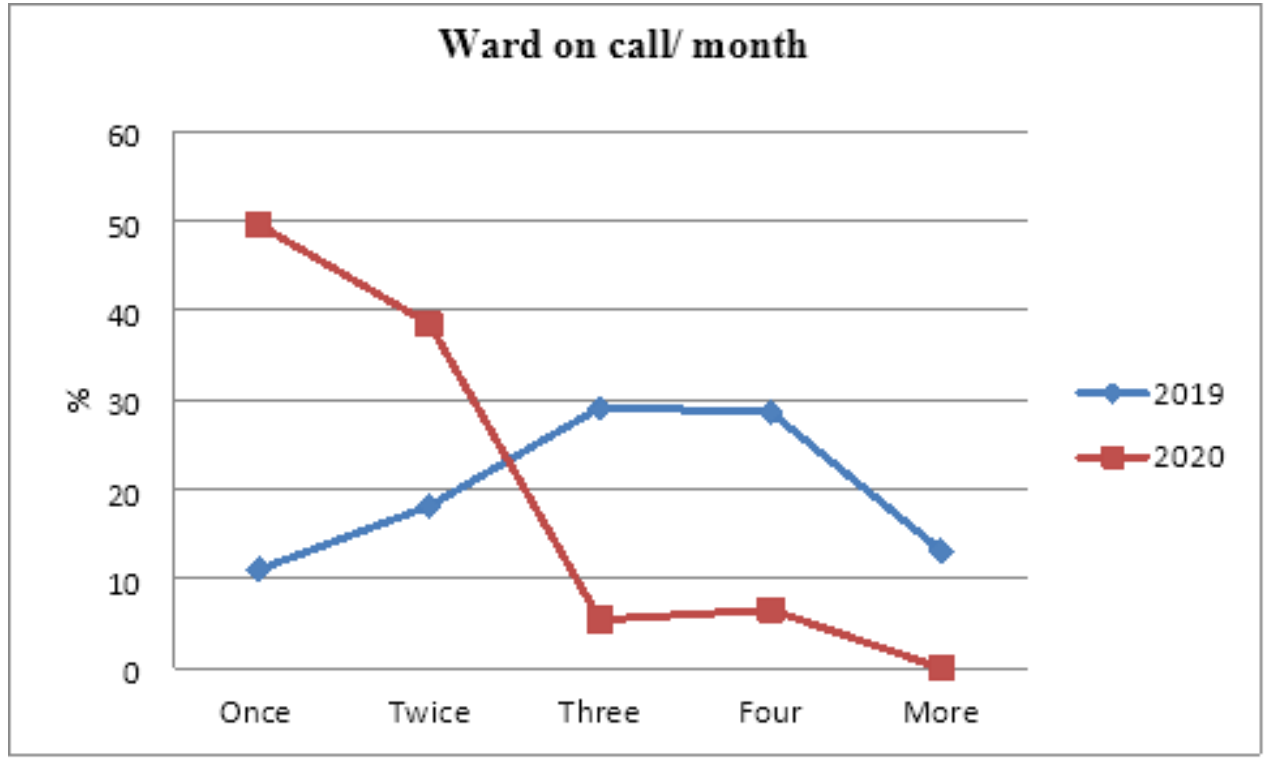

Figure 2

Trainees' Ward on call per month in 2019 compared to 2020 


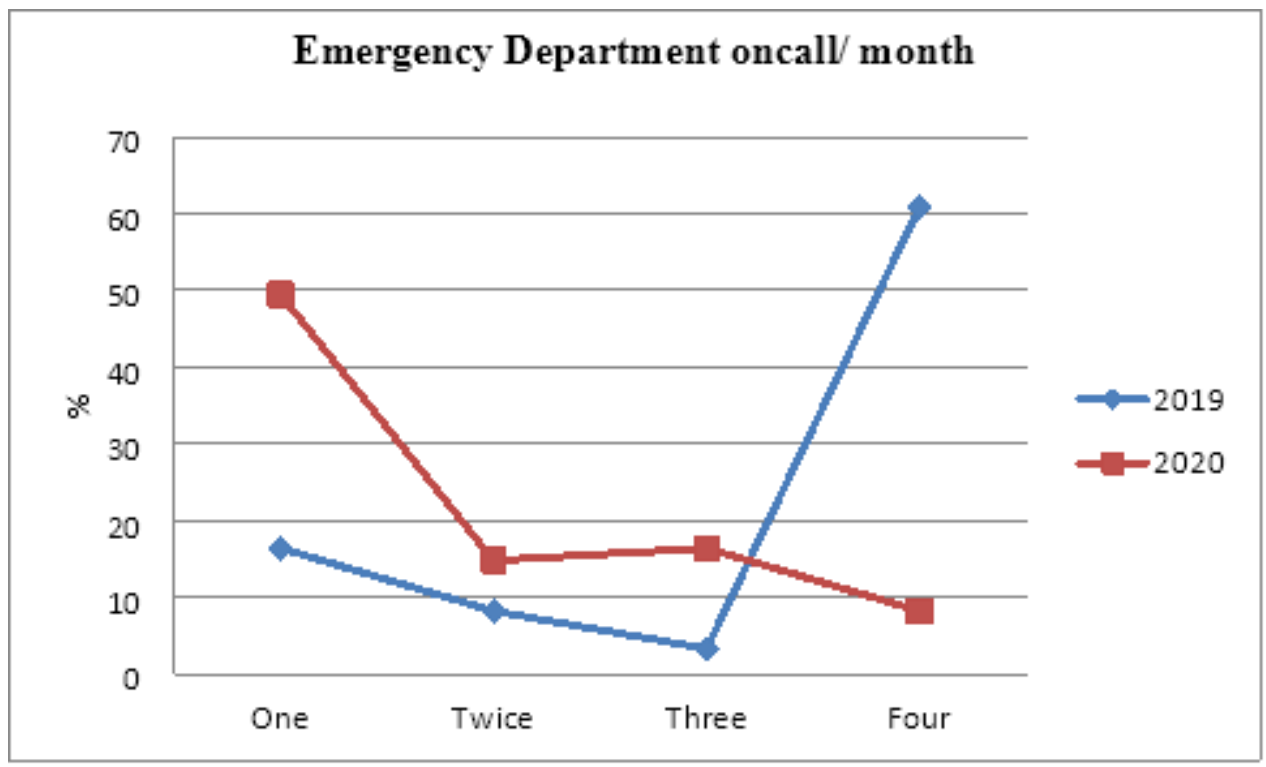

Figure 3

Trainees' emergency room on call per month in 2019 compared to 2020

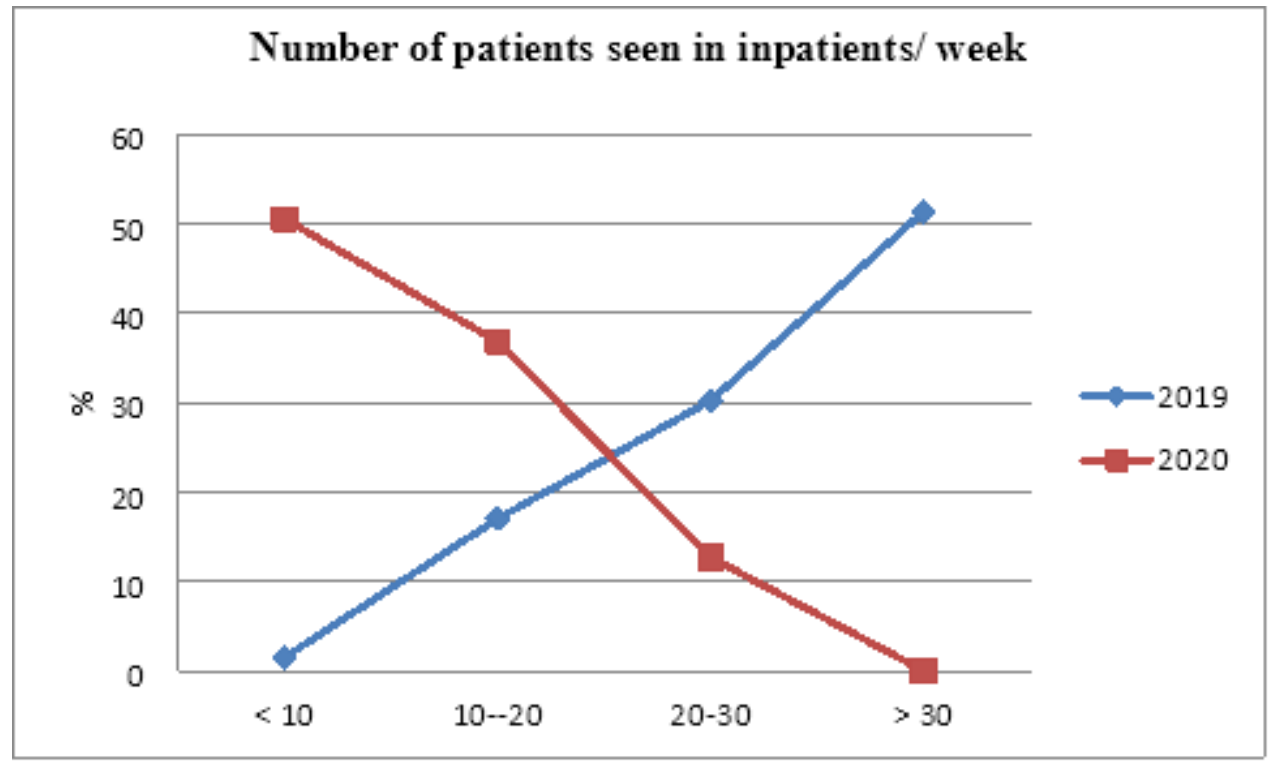

Figure 4

Number of patients seen in inpatient per week in the year2019 compared to 2020 


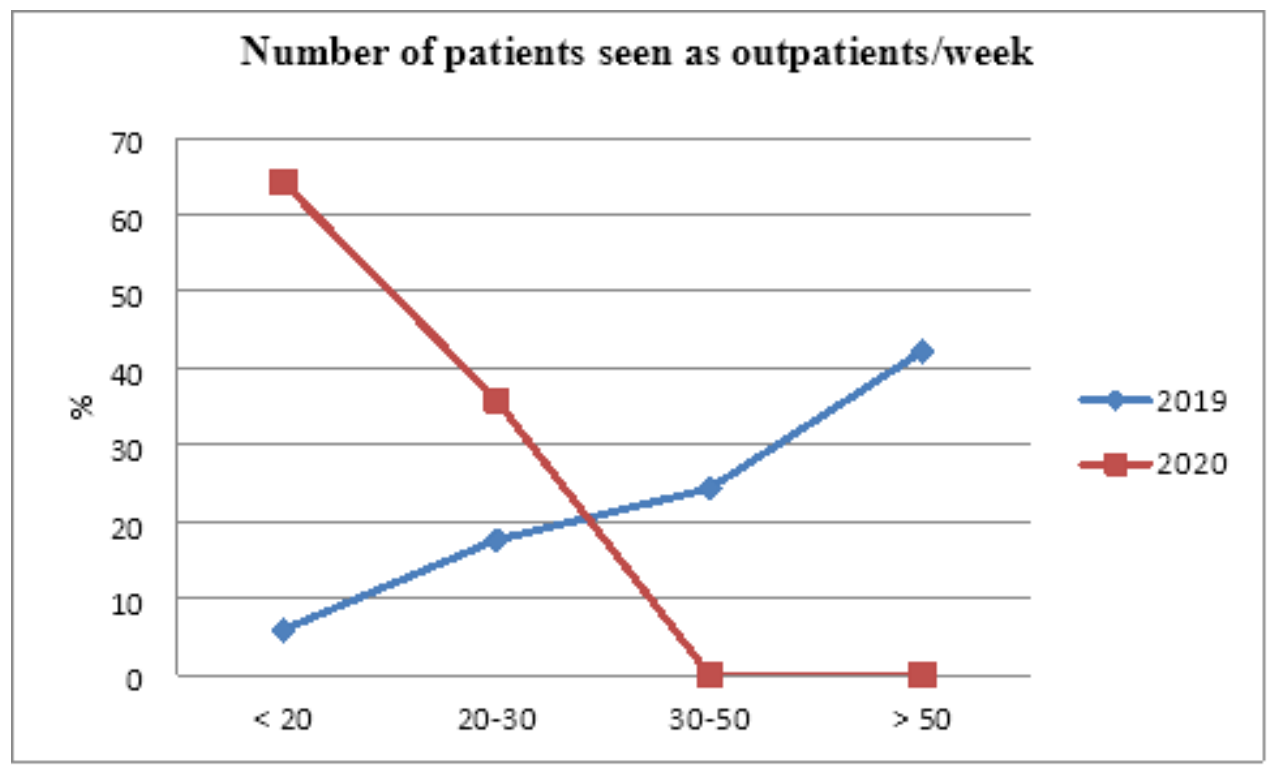

Figure 5

Number of patients seen in outpatient department per week in the year2019 compared to 2020

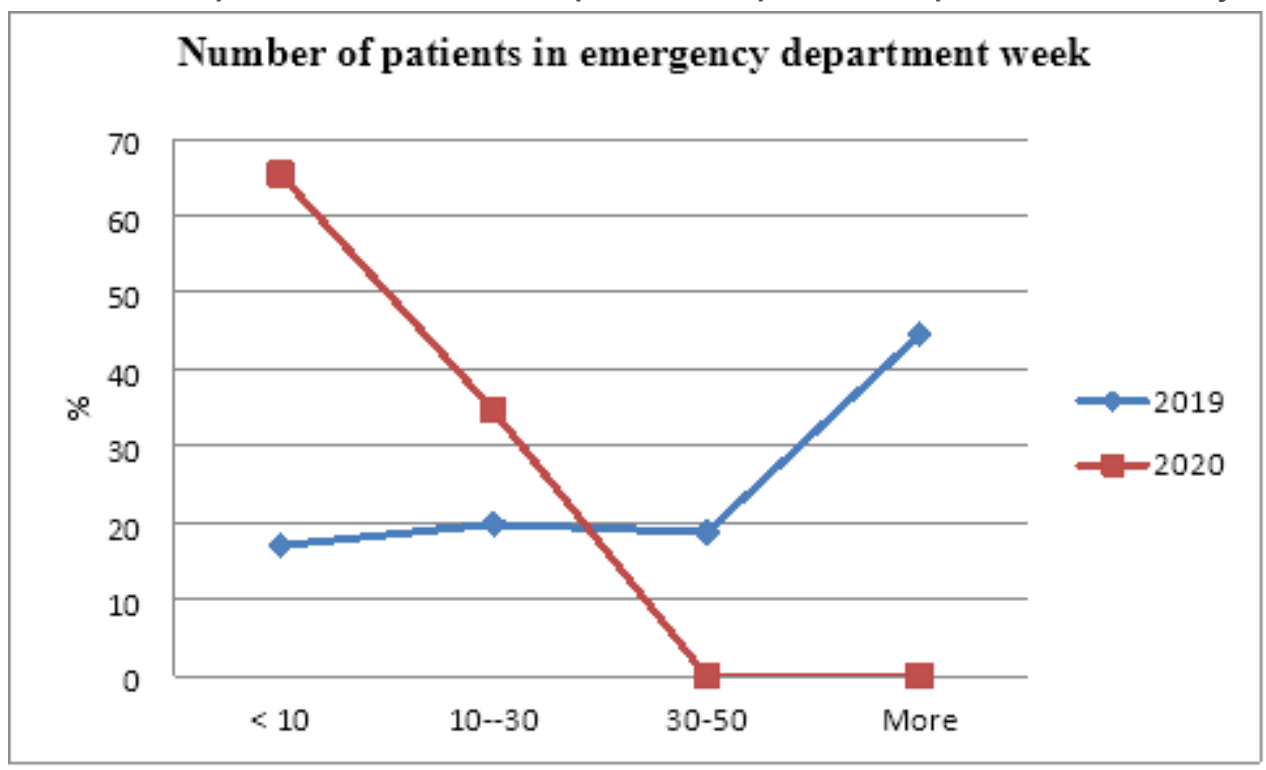

Figure 6

Number of patients seen at Emergency department per week in the year2019 compared to 2020 